\title{
Associações entre doença de Kawasaki e síndrome inflamatória multissistêmica em pacientes pediátricos com COVID-19
}

\author{
Associations between Kawasaki disease and multisystem inflammatory syndrome in \\ pediatric patients with COVID-19
}

\begin{abstract}
Asociaciones entre la enfermedad de Kawasaki y el síndrome inflamatorio multisistémico en pacientes pediátricos con COVID-19
\end{abstract}

\section{Cláudia Cássia Gama ${ }^{1 *}$, Isabelle Faria Safadi², Tainá Wendling Gama², Gabriela Araújo Costa².}

\section{RESUMO}

Objetivo: Abordar associações científicas estabelecidas até o momento entre a doença de Kawasaki e a Síndrome inflamatória multissistêmica associada a COVID-19 em pacientes pediátricos. Revisão bibliográfica: A doença de Kawasaki (DK) e a Síndrome Inflamatória Multissistêmica Pediátrica (SIM-P) possuem similaridades quanto aos aspectos clínicos e laboratoriais. Casos suspeitos de DK em pacientes pediátricos com Sars-CoV-2 foram posteriormente confirmados como SIM-P, mostrando a importância do profissional de saúde estar alerta às diferenças entre os quadros, que determinam diferentes condutas. Com isso, é importante destacar as principais complicações advindas das duas doenças e alertar sobre as consequências dessas intercorrências para a saúde, como choque com necessidade de suporte com inotrópicos; miocardite e insuficiência cardíaca, usualmente com necessidade de suporte respiratório e aminas vasopressoras. É necessário possibilitar diagnóstico e terapêutica precoces, que possibilitem melhor prognóstico, evitando futuras complicações de ambas as doenças em crianças e adolescentes. Considerações finais: Este artigo tem como objetivo diferenciar a SIM-P da DK, para que seja possível direcionar a terapia e a conduta correta para cada doença, proporcionando melhores prognósticos.

Palavras-chave: Doença de Kawasaki, Síndrome inflamatória multissistêmica, Infecção pelo Sars-CoV-2.

\begin{abstract}
Objective: Approach scientific associations established until this moment about Kawasaki disease and Multisystem Inflammatory Syndrome associated with COVID-19 in pediatric patients. Bibliographic review: Kawasaki disease (KD) and Multisystem Inflammatory Syndrome (MISC) have similarities when the topic is clinic and laboratory aspects. Suspicious cases of DK in pediatrics patients with Sars-CoV-2 were confirmed as MISC afterwards, demonstrating the importance of all professionals of health to knowing the differences between both diseases, which determines different conducts. Thus, it's important to highlight the main complications arising from the two diseases and warn about the consequences of these complications for health, as shock in need of support with inotropics; myocarditis and heart failure, usually requiring respiratory support and vasopressor amines. It's necessary to give a diagnosis and a treatment at the right moment for a better prognosis, avoiding further complications of both diseases in children and adolescents. Final considerations: This article aims to differentiate SIM-P from KD, so that it is possible to direct the therapy and the correct conduct for each disease, providing better prognosis.
\end{abstract}

Keywords: Kawasaki disease, Multisystem inflammatory syndrome, Infection by Sars-CoV-2.

\footnotetext{
1 Pontifícia Universidade Católica de Minas Gerais (PUC MINAS), Belo Horizonte - MG.

*E-mail: gamac.claudia@gmail.com

${ }^{2}$ Centro Universitário de Belo Horizonte (UNIBH), Belo Horizonte - MG.
} 


\section{RESUMEN}

Objetivo: Abordar las asociaciones científicas basadas hasta ahora entre la enfermedad de Kawasaki y un síndrome inflamatorio multisistémico asociado con un COVID-19 en pacientes pediátricos. Revisión bibliográfica: La enfermedad de Kawasaki (KD) y el síndrome inflamatorio multisistémico pediátrico (P-SIM) tienen similitudes en los aspectos clínicos y de laboratorio. Los casos sospechosos de en pacientes pediátricos con Sars-CoV-2 se confirmaron posteriormente como SIM-P, lo que demuestra la importancia de que el profesional de la salud conozca las diferencias entre las condiciones, que condicionan los diferentes abordajes. Así, es importante destacar como principales complicaciones derivadas de las dos enfermedades y advertir sobre las consecuencias de estas complicaciones para la salud, como el shock con la necesidad de apoyo con agentes inotrópicos; miocarditis e insuficiencia cardíaca, que generalmente requieren apoyo respiratorio y aminas vasopresoras. Es necesario posibilitar un diagnóstico y una terapia precoces, que permitan un mejor pronóstico, evitando futuras complicaciones de ambas enfermedades en niños y adolescentes. Consideraciones finales: Este artículo tiene como objetivo diferenciar SIM-P de KD, de manera que sea posible orientar la terapia y la conducta correcta para cada enfermedad, proporcionando un mejor pronóstico.

Palabras clave: Enfermedad de Kawasaki, Síndrome inflamatorio multisistémico, Infección por Sars-CoV-2.

\section{INTRODUÇÃO}

A doença do novo coronavírus, em inglês denominado coronavírus disease 2019 (COVID-19), responsável por casos de síndrome respiratória aguda grave (Sars-CoV-2) teve seu primeiro caso registrado na cidade de Wuhan, na China, em 31 de dezembro de 2019. Em seguida, diante da sua velocidade de propagação e transmissibilidade, a pandemia do novo coronavírus foi reconhecida pela Organização Mundial da Saúde (OMS) no dia 11 de março de 2020 (LANA RM, et al., 2020).

O primeiro caso da doença relatado no Brasil foi em fevereiro de 2020. Desde então, há um incremento das notificações e óbitos pelo país, alcançando números alarmantes. Uma importante questão epidemiológica é a elevada infectividade do Sars-CoV-2, cuja velocidade de propagação pode variar de 1,6 a 4,1 (isso significa que, cada 100 infectados podem transmitir a comorbidade para outras 16 a 41 pessoas) (MALTA DC, et al., 2020).

Devido à escassez de ações preventivas e um tratamento específico cientificamente comprovado para COVID-19, bem como seu impacto na morbimortalidade populacional, a OMS recomendou a adoção por parte dos governos, de práticas de cunho individual, como o incentivo ao uso de máscaras e lavagem rotineira das mãos, de cunho comunitário, como a restrição de horário ou proibição do funcionamento de ambientes passíveis de aglomeração, e de cunho ambiental, englobando protocolos de higienização dos locais (MALTA DC, et al., 2020).

Acredita-se que a maioria dos casos de COVID-19 na faixa etária pediátrica sejam leves ou assintomáticos. Entre os sintomáticos, é prevalente a ocorrência de febre, tosse, coriza, mialgia, vômitos e diarreia. Não é usual a ocorrência de casos graves com necessidade de internação em unidades de terapia intensiva ou que possuem o óbito como desfecho (GUIMARÃES AC, et al., 2020).

Moura SK, et al. (2020) mais recentemente, relatam séries de casos graves em crianças ou adolescentes, previamente hígidos, que apresentam uma síndrome inflamatória multissistêmica após a infecção pelo coronavírus. A Síndrome Inflamatória Multissistêmica Pediátrica (SIM-P) é caracterizada por febre, exantema, hiperemia conjuntival, edema nas extremidades, mucosite e cefaléia, entre outros. A sintomatologia da síndrome se assemelha à Doença de Kawasaki (DK), por isso, em um primeiro momento a SIM-P foi descrita como DK. Todavia, a SIM-P acomete geralmente crianças mais velhas e adolescentes, manifesta-se com sintomas gastrointestinais, disfunção miocárdica e choque, diferentemente do que habitualmente ocorre na DK. À medida em que novos casos da SIM-P foram relatados, foi possível distinguir ambas doenças e definir os critérios de diagnóstico para a SIM-P. 
A DK é uma doença já descrita e possui grande relevância clínica por ser causa importante de cardiopatia adquirida em crianças. É caracterizada por uma inflamação aguda de vasos sanguíneos, com manifestações sistêmicas e sem etiologia elucidada. Devido às suas características clínico-epidemiológicas, acredita-se que um agente infeccioso seja responsável pelo desenvolvimento da doença. Devido à alta transmissibilidade e infectividade, o novo Coronavírus pode representar um risco maior para doença de Kawasaki, considerandose a hipótese do gatilho viral para desencadear a síndrome (PRUC M, et al., 2020).

A DK envolve febre com duração superior a cinco dias, conjuntivite bilateral sem exsudato, eritema, enantema, exantema polimorfo, tosse, diarréia, alteração na cavidade oral, alteração nas extremidades e adenomegalia cervical. Em alguns casos, a apresentação clínica pode ser atípica, assemelhando-se ao quadro clínico da SIM-P. Por isso, os profissionais de saúde devem considerar a possibilidade de ambas as doenças (SIM-P e DK) em qualquer criança ou adolescente com infecção prévia por Sars-CoV-2 suspeita ou confirmada e que apresente os critérios diagnósticos que as duas patologias compartilham (LESSA AR, et al., 2021; BEREZIN E, 2020).

De acordo com Pacífico DK (2020), além das diferenças no quadro clínico, a DK ocorre com maior frequência em crianças com idade inferior a cinco anos, o que pode auxiliar no diagnóstico diferencial com a SIM-P A fisiopatologia dessa doença baseia-se na destruição da lâmina elástica interna dos vasos e, com isso, uma eventual proliferação de fibroblastos pode ocorrer de forma tardia. Ademais, ocorre um defeito na estrutura do vaso, contribuindo para uma maior fragilidade com consequente formação de aneurisma. Segundo Alonso IAF et al. (2021), na SIM-P, acredita-se que uma reação hiper inflamatória desequilibrada, mediada pela imunidade inata, seja responsável pela agressão tecidual, incluindo o estado hiper inflamatório do ventrículo esquerdo.

Conforme Linhares MBM e Enumo SRF (2020), o primeiro caso registrado de SIM-P no mundo foi em abril de 2020, nos Estados Unidos, em uma criança com seis meses de vida com sintomas semelhantes à DK após infecção pelo COVID-19; desde então, multiplicaram-se relatos de quadros semelhantes na Europa, Estados Unidos e vários países da América Latina. Neves U (2021) afirma que, segundo informações da OMS, o Brasil contabilizou mais de 700 casos e aproximadamente 50 óbitos de pacientes pediátricos pela SIM-P associada a Covid-19, de abril de 2020 a fevereiro de 2021.

É importante realçar que a SIM-P não ocorre concomitante ao quadro de infecção aguda pelo Sars-Cov 2 e sim após uma média de 14 dias, o que reforça a hipótese de ocorrer por resultado de uma disfunção de resposta imune ao vírus. Os pacientes apresentam, após um período variável de infecção pelo SARS-Cov 2, febre alta persistente e manifestações gastrointestinais exuberantes em 50-60\% dos casos (dor abdominal intensa, diarréia e vômitos), algumas vezes com evolução para choque (manifesto por hipotensão arterial, taquicardia e distúrbio de perfusão) (CARVALHO H, et al., 2020; CAMPOS L, et al., 2020).

Os sintomas respiratórios costumam ser menos frequentes e relevantes, muitas vezes atribuídos à descompensação hemodinâmica e não a comprometimento pulmonar direto. Não existe um protocolo único válido para o tratamento de todos os casos da SIM-P; todavia, indica-se o uso de Imunoglobulina endovenosa e ácido acetilsalicílico para casos que se enquadrem nos critérios diagnósticos da DK clássica (CAMPOS L, et al., 2020).

A partir da análise das similaridades entre a SIM-P e a DK é possível compreender as principais diferenças entre ambas as doenças e, com isso, a conduta médica, a propedêutica e os métodos de tratamento utilizados para cada caso podem ser mais adequados e, dessa forma, com melhor prognóstico. Destacar a fisiopatologia de cada patologia é necessária para uma ação mais rápida e mais precisa, evitando complicações graves e, até mesmo, fatais.

\section{REVISÃO BIBLIOGRÁFICA}

A Doença de Kawasaki (DK) acomete, comumente, crianças menores de cinco anos de idade. Trata-se de uma vasculite inflamatória aguda que atinge pequenos e médios vasos, de etiologia ainda desconhecida. Cursa com manifestações inflamatórias sistêmicas, geralmente autolimitadas, mas que podem, enquanto ativas, elevarem o risco de óbito e de sequelas, tais como os aneurismas de artérias coronárias, constituindose em uma das principais causas de doença cardíaca adquirida no mundo (RODRIGUES M, et al., 2018). 
Outra doença muito semelhante a DK vem sendo recentemente relatada na literatura, associada à pandemia da COVID-19. Denominada síndrome inflamatória multissistêmica, apresenta sintomas muito parecidos com a doença de Kawasaki, porém com algumas diferenças, como faixa etária de maior incidência, alta prevalência de manifestações gastrointestinais e neutrofilia significativa. Como na DK, alterações cardiológicas podem ser encontradas, embora estejam mais associadas a miocardite e insuficiência cardíaca e não ao acometimento coronariano. O reconhecimento precoce da síndrome inflamatória multissistêmica associada ao Sars-CoV-2 e, consequentemente, a rápida intervenção, com especial atenção à identificação da necessidade de cuidados intensivos, são cruciais para evitar óbitos nas crianças acometidas (SÁFADI MAP, 2020).

É necessário destacar o fato de que até a chegada do Sars-CoV-2 no Brasil, o protocolo de vigilância de Síndrome Respiratória Aguda Grave não incluía o novo coronavírus como um patógeno de destaque no painel de exame laboratorial, com exceção para o estado do Paraná. Além disso, é importante destacar que o setor responsável por dados epidemiológicos no Brasil encontra obstáculos como a desigualdade de investimento em sistemas de análise em tempo real e diferenças nos sistemas de vigilância em saúde, ainda que existam políticas de transparência como o Sistema Eletrônico do Serviço de Informações ao Cidadão (e-SIC) (LANA RM, et al., 2020).

Sabe-se também que dados como o número de hospitalizações, bem como as taxas de mortalidade entre os doentes, influenciam no desenvolvimento de medidas preventivas e planos de enfrentamento da COVID19 no território brasileiro. Uma atenção especial é dada para as crianças e adolescentes uma vez que, embora não sejam os mais afetados, possuem importância clínica por serem grandes transmissores da doença, além de apresentarem aumento da letalidade principalmente por causa da síndrome inflamatória multissistêmica (HILLESHEIM D, et al., 2020).

De acordo com os critérios estabelecidos pela American Heart Association (AHA) e pela European League Against Rheumatism/Pediatric Rheumatology European Society (EULAR/PReS), o diagnóstico da doença de Kawasaki é essencialmente clínico e envolve: febre persistente sem foco por pelo menos cinco dias, associada a quatro ou mais dos seguintes critérios: alteração de lábios e cavidade oral, tais como eritema e fissuras labiais e/ou enantema orofaríngeo e/ou "língua em framboesa ou em morango", sem lesões ulceradas, além de hiperemia conjuntival bilateral e sem exsudato, alteração de extremidades ( edema, eritema e/ou descamação em dorso), exantema polimorfo e linfadenopatia cervical $\geq 1,5 \mathrm{~cm}$, usualmente unilateral (SILVA C, et al., 2019).

Dentre os achados laboratoriais, destacam-se o aumento dos marcadores inflamatórios Velocidade de Hemossedimentação (VHS) e Proteína C reativa (PCR), hiponatremia, leucocitose com neutrofilia acompanhada de basto anemia hipoalbuminemia, aumento moderado de transaminases, anemia normocítica normocrômica, trombocitose e piúria estéril. Os pacientes que não se enquadram nos critérios diagnósticos são classificados como portadores de DK atípica ou incompleta. Deve-se suspeitar de DK incompleta quando há a presença de febre inexplicada ( 5 dias ou mais) associada a dois ou mais critérios clínicos estabelecidos para diagnóstico da doença (RODRIGUES M, et al., 2018; ALONSO IAF, et al., 2021).

Na síndrome inflamatória multissistêmica, os sintomas são, basicamente, febre, diarréia e dor abdominal, rash cutâneo, vômitos, cefaléia, sintomas respiratórios secundários ao acometimento cardiológico e, em uma significativa parcela dos pacientes pediátricos, choque. As alterações laboratoriais, indicam linfopenia, neutrofilia, aumento de ferritina, dímero $D$, proteína $C$ reativa e troponina, além de anemia e plaquetopenia (BEREZIN E, 2020).

Segundo Berezin E (2020), o desfecho clínico, na maioria dos casos, são: choque com necessidade de suporte com inotrópicos; miocardite e insuficiência cardíaca, usualmente com necessidade de suporte respiratório e aminas vasopressoras. A realização de swab nasal para identificação da infecção pelo SarsCoV-2 não está indicada nessa fase, visto que a SIM-P, tipicamente, manifesta-se após, em média, 14 dias da infecção, quando este exame, habitualmente, já estará negativo. Todavia, uma grande parcela de indivíduos na faixa etária pediátrica com SIMP-P apresentou teste positivo para Covid-19, indicando uma infecção recém-adquirida. 
Farias ECF, et al. (2020) propõe-se que a infecção por Sars-CoV-2 evolua em três fases, causando maior mortalidade na terceira, após duas semanas ou mais do início dos sintomas. Uma minoria de pacientes alcançará a terceira fase de tempestade de citocinas inflamatórias. A síndrome pode surgir sem que a criança tenha manifestado, previamente, qualquer sinal da infecção por covid-19, visto que, nas crianças, é muito comum que a infecção pelo coronavírus apresente-se de forma oligo ou assintomática. Cerca de três quartos das crianças acometidas por SIM-P nos Estados Unidos não apresentaram sintomas de covid-19 em sua história pregressa (BEREZIN E, 2020).

Campos LR, et al. (2019) afirma que é possível a análise dos critérios para o diagnóstico da síndrome inflamatória multissistêmica pediátrica, baseando-se na gravidade da doença, nos critérios para doença de Kawasaki e além desses sinais e sintomas, baseando-se também na propedêutica a ser realizada de acordo com o caso (Figura 1). Em crianças e adolescentes entre 0-21 anos, é preciso observar se os critérios preenchem para DK ou para síndrome do choque tóxico ou se a febre for superior ou igual a $38^{\circ} \mathrm{C}$ por no mínimo 3 dias, além de 1 dos seguintes fatores: sintomas do TGI, sintomas neurológicos, rash difuso, conjuntivite, alteração da mucosa oral, alterações de extremidades e linfonodomegalia cervical.

Caso não preencha nenhum dos critérios acima, a probabilidade é baixa de ser SIMP. Caso preencha algum dos critérios, ver se existe algum de gravidade, como choque, hipotensão ou disfunção cardíaca, perfusão lentificada, alteração do nível de consciência, taquipnéia ou dispnéia, estertores, ritmo de galope e arritmia. Se não preencher os critérios de gravidade ou se preencher, observar se é similar aos sintomas de DK, caso preencha, solicitar painel estendido de exames complementares, acionar especialistas e caso não seja possível contatar especialista, tratar (CAMPOS LR, et al., 2019).

Se não preencher os critérios para DK, solicitar exames laboratoriais e se o foco for identificado a probabilidade de ser SIMP é baixa, mas se não for identificado foco, observar se o valor do exame PCR está elevado, ou seja, maior que $3 \mathrm{mg} / \mathrm{dl}$, para que então sejam realizados mais exames complementares (CAMPOS LR, et al., 2019).

Figura 1 - Fluxo para o diagnóstico da SIM-P.

Crianças e adolescente entre 0 e 21 anos apresentando critérios para DK OU síndrome do choque tóxico OU febre maior ou igual a $38^{\circ} \mathrm{C}$ por 3 dias ou mais com mais de um dos fatores abaixo:

\author{
Sintomas do TGI \\ Sintomas neurológicos \\ Conjuntivite \\ Rash difuso \\ Alteração da mucosa oral \\ Alterações de extremidades, como edema \\ Linfonodomegalia cervical
}

Observar se além dos sintomas acima, se existem sinais de gravidade: choque, hipotensão, disfunção cardiaca, perfusão lentificada, alteração do nível de consciência, taquipnéia, dispnéia, estertores, ritmo de galope e arritmia.

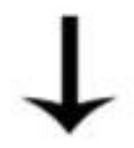

Solicitar painel estendido de exames complementares e acionar especialistas

Fonte: Gama CC, et al., 2021; baseado em: Campos LR, et al., 2019; Berezin EN, 2020; Volpat AT, et al., 2020. 
É possível fazer uma comparação detalhada dos métodos diagnósticos entre SIM-P e DK. A doença de Kawasaki é analisada laboratorialmente por meio de: PCR, VHS, procalcitonina, fibrinogênio, D-dímero, ferritina, LDH e IL-6. Além destes métodos confirmatórios, é também sugerido a realização de exames complementares, como ecocardiograma, eletrocardiograma e a análise das enzimas cardíacas (CAMPOS LR, et al., 2019).

Em contrapartida, o diagnóstico da síndrome inflamatória multissistêmica se baseia em exames laboratoriais que possam descartar outras hipóteses diagnósticas, sendo necessário: hemograma, VHS, PCR, anticorpos antinucleares, fator reumatoide, albúmina, enzimas hepáticas, exame de urina. É também indicado a realização de exames complementares, como ECG seriada, ecocardiograma e radiografia do tórax. Notase, na maioria dos casos, um aumento dos níveis séricos de PCR, ferritina, dímero-D e fibrinogênio, caracterizando um estado inflamatório e pró-trombótico (FERREIRA BWRC, et al., 2021; BARSAM FJBG, et al., 2021).

Consoante com Silva C (2019), na avaliação por exames complementares, o ecocardiograma com Doppler deve ser realizado o mais precocemente possível, idealmente no momento da suspeita tanto da DK quanto da SIM-P, a fim de detectar ectasias, aneurismas, tortuosidades e estreitamentos das artérias coronárias em ambas as doenças. Outros exames complementares são necessários, como: hemograma completo, marcadores inflamatórios, Aspartato Aminotransferase (AST), Alanina Aminotransferase (ALT), GamaGlutamil Transferase (GGT), Fosfatase Alcalina (FA), Bilirrubina Total e frações (BT), proteínas totais e frações, uréia, creatinina, Elementos Anormais e Sedimentos da urina (EAS), amilase, lipase e triglicerídeos, Tempo de Atividade de Protrombina (TAP), Tempo de Tromboplastina Parcial (PTT) e fibrinogênio.

Silva C (2019) afirma que outros fatores são também incluídos para auxiliar no diagnóstico, como a presença de biomarcadores como a Creatinoquinase Miocárdica (CK-mb), Creatinoquinase (CK), pro-BNP (peptídeo natriurético tipo B) e a troponina. Além desses, RT-PCR, sorologia para Sars-CoV-2 e outras culturas e sorologias podem ser úteis. Campos L, et al. (2019) afirma que exames complementares como o Eletrocardiograma, a Ultrassonografia de abdome, a Tomografia Computadorizada e a Radiografía de tórax podem ser necessários. Diante disso, com a união da clínica com os achados laboratoriais e de imagem é possível chegar ao diagnóstico da SIM-P e da DK.

O tratamento da DK deve ser feito de forma precoce, com imunoglobulina humana intravenosa (IVIG) em dose única de $2 \mathrm{~g} / \mathrm{Kg}$, até o $10^{\circ}$ dia de febre. O objetivo é reduzir a incidência de lesão coronariana de $25 \%$ para inferior a 4\%. Todavia, na persistência da elevação de VHS ou PCR, associado a febre, a IVIG também deve ser administrada mesmo passados os 10 dias de início da febre. Devido ao risco de miocardite e arritmias, é recomendado manter o paciente internado para monitorização cardíaca (RODRIGUES M, et al., 2018).

Rodrigues $\mathrm{M}$, et al. (2018) recomenda-se também a associação de ácido acetilsalicílico (AAS) em doses moderadas (30 a 50mg/Kg/dia) ou altas (50 a $80 \mathrm{mg} / \mathrm{kg} / \mathrm{dia}$ ). O AAS apresenta efeito anti-inflamatório e antiagregante plaquetário, potencializando o efeito anti-inflamatório da IVIG.

Na síndrome inflamatória multissistêmica, todas as crianças que estiverem estáveis, sem sinais clínicos de gravidade, devem ser avaliadas em serviços com experiência no tratamento de doenças infecciosas e síndromes inflamatórias, para garantir tratamento imediato no caso de complicações. A identificação de critérios para encaminhamento à terapia intensiva deve ser a mais rápida possível, devido à alta frequência de choque descrito nessas crianças. Nestes casos, dados da literatura indicam administração de gamaglobulina endovenosa (BEREZIN EN, 2020).

Segundo Campos L, et al. (2020) Nos casos de SIM-P leves a moderados, alguns profissionais optam pelo uso de enoxaparina e em casos mais graves, com dose terapêutica, apesar de não haver consenso. A indicação de corticoide para todos os casos de SIM-P permanece controversa; a tendência atual é de utilizálo associado à imunoglobulina endovenosa nos casos graves, caracterizados por quadro inflamatório intenso com febre persistente, que pode levar à choque e falência de múltiplos órgãos, necessitando de internação hospitalar (MOURA SK, et al., 2020). 
Além disso, é importante considerar as terapias antivirais e imunomoduladoras somente em quadros clínicos definidos, além de ser avaliada entre os profissionais de saúde responsáveis junto dos comitês de ética. Alguns tratamentos incluem a administração de ácido acetilsalicílico (aspirina), podendo ser usado na dose de $80-100 \mathrm{mg} / \mathrm{kg} / \mathrm{dia}$ como anti-inflamatório não esteroidal no primeiro momento, alterando a dose 3$5 \mathrm{mg} / \mathrm{kg} / \mathrm{dia}$, após o cessamento do quadro febril. Além dos pacientes com SIM-P, aqueles correspondentes à DK completa ou incompleta devem iniciar as mesmas medidas de tratamento (CAMPOS L, et al., 2019).

Considera-se que exista interação do Sars-CoV-2 com a ECA2. Entretanto, é um assunto muito discutido ao envolver a população pediátrica uma vez que, quando comparado aos adultos, a enzima conversora de angiotensina 2 possui imaturidade, diferenças funcionais e morfológicas. Visto isso, o papel da ECA2 não foi totalmente esclarecido diante das interações de seu uso quando associada à comorbidade, podendo agravar o quadro clínico da doença em qualquer faixa etária (VOLPAT AT, et al., 2020).

Alguns desfechos clínicos podem ocorrer diante de um quadro de SIM-P. O mais comum das complicações é o choque com necessidade de suporte inotrópicos, correspondente a $70 \%$ dos casos, em seguida envolvimento do miocárdio (51\%), envolvimento de artéria coronária (13\%), necessidade do uso de ECMO oxigenação por membrana extracorpórea (3\%) e, por último, mas não menos recorrente, a necessidade de suporte respiratório (67\%) (BEREZIN EN, 2020).

\section{CONSIDERAÇÕES FINAIS}

Há uma relação entre a COVID-19 pediátrica e a SIM-P. Esta complicação da infecção pelo COVID-19 tem semelhanças clínicas com a DK, mas difere em aspectos como faixa etária, sintomas gastrointestinais e acometimento miocárdico. Devido a essa similaridade clínica e ambas poderem ser desencadeadas pelo coronavírus, destaca-se a necessidade de preparação dos profissionais de saúde para realizar o diagnóstico precoce e a intervenção para ambas as doenças, encaminhando os pacientes para instituições com experiência no manejo dessas. O desfecho dos pacientes com a síndrome inflamatória multissistêmica pode ser mais grave do que os da doença de Kawasaki, podendo evoluir, na maior parte dos casos, com necessidade de suporte respiratório e uso de inotrópicos.

\section{REFERÊNCIAS}

1. ALIZARGAR J. The novel coronavirus (COVID-19) and the risk of Kawasaki disease in children. Journal of the Formosan Medical Association, 2020; 119(11): 1713-1714.

2. ALONSO, IAF, et al. Doenças cardiovasculares em crianças com síndrome inflamatória multissistêmica decorrente de infecção por covid-19: uma revisão integrativa. Brazilian Journal of Development, 2021; 7:7.

3. ALVIM ALS, et al. Síndrome inflamatória multissistêmica em crianças e adolescentes com COVID-19: uma revisão de literatura. Journal infection control, 2020; (9): 3.

4. BARSAM, FJBG, et al. Manejo da Síndrome Inflamatória Multissistêmica Pediátrica, MG. Protocolo clínico, Universidade Federal do Triângulo Mineiro Hospital das Clínicas, 2021; 14p.

5. BEREZIN EN. Síndrome inflamatória multissistêmica pediátrica (síndrome associada temporalmente ao COVID-19). Sociedade de Pediatria de São Paulo, 2020; 4p.

6. CAMPOS LR, et al. Síndrome inflamatória multissistêmica pediátrica (MIS-C) temporariamente associada ao SarsCov-2. Residência Pediátrica- a Revista do Pediatra, 2020; 10(2): 1-6.

7. CARVALHO HT, et al. Manifestações graves da Doença de Kawasaki em tempos de COVID-19: relato de caso. Revista Residência Pediátrica, 2020; 2020;10(3): 1-5.

8. GUIMARÃES AC, et al. COVID-19 in children: considerations for returning to school. Braz J Otorhinolaryngol. 2020; 86: 667-8.

9. HILLESHEIM D, et al. Síndrome respiratória aguda grave por COVID-19 em crianças e adolescentes no Brasil: perfil dos óbitos e letalidade hospitalar até a 38a Semana Epidemiológica de 2020, 2020; 29(5), 1 p.

10. LANA RM, et al. Emergência do novo coronavírus (SARS-CoV-2) e o papel de uma vigilância nacional em saúde oportuna e efetiva, 2020; 29 (5).

11. LESSA AR, et al. Associação de SARS-COV-2 com a doença de Kawasaki. Revista Eletrônica Acervo Científico, 2021; 23: e6462.

12. LINHARES MM, ENUMO SRF. Reflexões baseadas na Psicologia sobre efeitos da pandemia COVID-19 no desenvolvimento infantil, 2020; 37: e200089. 
13. MALTA DC, et al. A pandemia da COVID-19 e as mudanças no estilo de vida dos brasileiros adultos: um estudo transversal, 2020, Epidemiol. Serv. Saúde, 2020; 29(4): e2020407.

14. MOURA SK, et al. Síndrome Inflamatória Multissistêmica Pediátrica. TelessaúdeRS, 2020.

15. PACÍFICO D, et al. Doença de Kawasaki e COVID-19: uma revisão de literatura. Revista Eletrônica Acervo Saúde, 2020; 12: e5058.

16. PRUC M, et al. Kawasaki disease shock syndrome or toxic shock syndrome in children and the relationship with COVID19. Journal Elsevier, 2020; 144: 109986.

17. RODRIGUES M, et al. Doença de Kawasaki e complicações cardiovasculares em Pediatria. Birth And Growth Medical Journal, 2018; 27:1.

18. SANTOS BS, et al. Relação clínico-epidemiológica entre Sars-Cov 2 e Doença de Kawasaki: uma revisão integrativa da literatura. Revista Paulista de Pediatria, 2020; 39: 1-3.

19. SÁFADI MAP. Síndrome inflamatória multissistêmica em crianças e adolescentes provavelmente associada à COVID19: uma apresentação aguda, grave e potencialmente fatal. Sociedade Brasileira de Pediatria, 2020; 1-3.

20. SILVA C, et al. Doença de Kawasaki. Departamento Científico de Reumatologia - Sociedade Brasileira de Pediatria, 2019; (6): 1-4.

21. FARIAS ECF. Síndrome Inflamatória Multissistêmica em criança associada a doença do Coronavírus 19 na amazônia brasileira: evolução fatal em lactente: Multisystem inflammatory syndrome in a child associated with Coronavirus disease 19 in the brazilian Amazon: fatal outcome in an infant, 2020; 38: e2020165.

22. FERREIRA BWRC. Síndrome Inflamatória Multissistêmica Pediátrica (SIM-P) temporariamente associada à COVID19: um levantamento das características clínicas e epidemiológicas, 2021; e5710313020. 